\title{
AN IDENTITY RELATED TO JORDAN'S INEQUALITY
}

\author{
JIAN-LIN LI
}

Received 28 July 2006; Revised 29 September 2006; Accepted 2 October 2006

The main purpose of this note is to establish an identity which states that the function $\sin x / x$ is a power series of $\left(\pi^{2}-4 x^{2}\right)$ with positive coefficients for all $x \neq 0$. This enable us to obtain a much stronger Jordan's inequality than that obtained before.

Copyright (c) 2006 Hindawi Publishing Corporation. All rights reserved.

\section{Introduction}

The well-known Jordan's inequality states that

$$
\frac{2}{\pi} \leq \frac{\sin x}{x}<1, \quad x \in\left(0, \frac{\pi}{2}\right]
$$

with equality holds if and only if $x=\pi / 2$ (see [5]). It plays an important role in many areas of pure and applied mathematics. The inequality (1.1) is first extended to the following:

$$
\frac{2}{\pi}+\frac{1}{12 \pi}\left(\pi^{2}-4 x^{2}\right) \leq \frac{\sin x}{x}<1, \quad x \in\left(0, \frac{\pi}{2}\right]
$$

and then, it is further extended to the following:

$$
\frac{2}{\pi}+\frac{1}{\pi^{3}}\left(\pi^{2}-4 x^{2}\right) \leq \frac{\sin x}{x}<1, \quad x \in\left(0, \frac{\pi}{2}\right]
$$

with equality holds if and only if $x=\pi / 2$ (see $[2,4,6]$ ). The inequality (1.3) is slightly stronger than the inequality (1.2) and is sharp in the sense that $1 / \pi^{3}$ cannot be replaced by a larger constant. More recently, the monotone form of L'Hopital's rule (see [1, Lemma 5.1]) has been successfully used by Zhu $[9,10]$, Wu and Debnath $[7,8]$ in the sharpening 
2 An identity related to Jordan's inequality

Jordan's inequality. For example, it has been shown that if $0<x \leq \pi / 2$, then

$$
\begin{gathered}
\frac{2}{\pi}+\frac{1}{\pi^{3}}\left(\pi^{2}-4 x^{2}\right) \leq \frac{\sin x}{x} \leq \frac{2}{\pi}+\frac{\pi-2}{\pi^{3}}\left(\pi^{2}-4 x^{2}\right) \\
\frac{2}{\pi}+\frac{1}{\pi^{3}}\left(\pi^{2}-4 x^{2}\right)+\frac{12-\pi^{2}}{16 \pi^{5}}\left(\pi^{2}-4 x^{2}\right)^{2} \leq \frac{\sin x}{x} \leq \frac{2}{\pi}+\frac{1}{\pi^{3}}\left(\pi^{2}-4 x^{2}\right)+\frac{\pi-3}{\pi^{5}}\left(\pi^{2}-4 x^{2}\right)^{2}
\end{gathered}
$$

hold with equality if and only if $x=\pi / 2$. Furthermore, the constants $1 / \pi^{3}$ and $(\pi-2) / \pi^{3}$ in (1.4) as well as the constants $\left(12-\pi^{2}\right) /\left(16 \pi^{5}\right)$ and $(\pi-3) / \pi^{5}$ in (1.5) are the best. Also, in the process of sharpening Jordan's inequality, one can use the same method as did in [7] to introduce a parameter $\theta(0<\theta \leq \pi)$ to replace the value $\pi / 2$. Unfortunately, the preceding method will become cumbersome to execute in the further generalization of Jordan's inequality.

In this note we establish an identity which states that the function $\sin x / x$ is a power series of $\left(\pi^{2}-4 x^{2}\right)$ with positive coefficients for all $x \neq 0$. This enables us to obtain a much better inequality than (1.4) or (1.5) if $0<x \leq \pi / 2$.

\section{Main result}

The main result relating to Jordan's inequality is contained in the following.

Theorem 2.1. For any $x>0$, the following identity

$$
\frac{\sin x}{x}=\frac{2}{\pi}+\sum_{k=1}^{\infty} \frac{(-1)^{k} R_{k}}{k ! \pi^{2 k}}\left(\pi^{2}-4 x^{2}\right)^{k}
$$

holds, where

$$
R_{k}=\sum_{n=k}^{\infty} \frac{(-1)^{n} n !}{(2 n+1) !(n-k) !}\left(\frac{\pi}{2}\right)^{2 n}
$$

satisfying $(-1)^{k} R_{k}>0(k=1,2,3, \ldots)$.

Proof. Let $x=\sqrt{\pi^{2}-t} / 2$ and $t=\pi^{2}-4 x^{2}$. It follows from the Taylor expansion for $\sin x$ that

$$
\frac{\sin \sqrt{\left(\pi^{2}-t\right)} / 2}{\sqrt{\left(\pi^{2}-t\right)} / 2}=\sum_{n=0}^{\infty} \frac{(-1)^{n}}{(2 n+1) !}\left(\frac{\pi^{2}-t}{4}\right)^{n} \quad\left(-\infty<t<\pi^{2}\right)
$$

Since

$$
\left(\pi^{2}-t\right)^{n}=\pi^{2 n}+\sum_{k=1}^{n} \frac{(-1)^{k} n !}{k !(n-k) !} \pi^{2(n-k)} t^{k} \quad(n=1,2,3, \ldots)
$$


we see that

$$
\begin{aligned}
\frac{\sin \sqrt{\left(\pi^{2}-t\right)} / 2}{\sqrt{\left(\pi^{2}-t\right)} / 2} & =1+\sum_{n=1}^{\infty} \frac{(-1)^{n}}{(2 n+1) !}\left(\frac{\pi}{2}\right)^{2 n}+\sum_{n=1}^{\infty} \frac{(-1)^{n}}{(2 n+1) ! 4^{n}} \sum_{k=1}^{n} \frac{(-1)^{k} n !}{k !(n-k) !} \pi^{2(n-k)} t^{k} \\
& =\frac{2}{\pi}+\sum_{k=1}^{\infty}\left\{\sum_{n=k}^{\infty} \frac{(-1)^{n}}{(2 n+1) !} \frac{n !}{(n-k) !}\left(\frac{\pi}{2}\right)^{2 n}\right\} \frac{(-1)^{k}}{k ! \pi^{2 k}} t^{k} \\
& =\frac{2}{\pi}+\sum_{k=1}^{\infty} \frac{(-1)^{k} R_{k}}{k ! \pi^{2 k}} t^{k} \quad\left(-\infty<t<\pi^{2}\right)
\end{aligned}
$$

which yields (2.1) with $R_{k}$ given by (2.2).

Let $R_{k}=\sum_{n=k}^{\infty}(-1)^{n} c_{n, k}$ with

$$
c_{n, k}=\frac{1}{(n-k) !} \frac{1}{n+1} \frac{\pi^{2} / 4}{n+2} \frac{\pi^{2} / 4}{n+3} \cdots \frac{\pi^{2} / 4}{n+n} \frac{\pi^{2} / 4}{2 n+1}
$$

Then for each given $k(k=1,2,3, \ldots)$,

$$
c_{n, k}>c_{n+1, k}, \quad(n \geq k), \quad \lim _{n \rightarrow \infty} c_{n, k}=0 .
$$

Hence the alternating series $\sum_{n=k}^{\infty}(-1)^{n} c_{n, k}$ converges, and its sum

$$
R_{k}=(-1)^{k} \sum_{j=0}^{\infty}(-1)^{j} c_{k+j, k}
$$

satisfies $(-1)^{k} R_{k}>0$ and $\left|R_{k}\right|<c_{k, k}$ for each given $k(k=1,2,3, \ldots)$. This completes the proof of the theorem.

Next we give a formula of calculating $R_{k}$. Let

$$
d_{k}(x)=\sum_{n=k}^{\infty} \frac{(-1)^{n} n !}{(2 n+1) !(n-k) !} x^{2 n}
$$

Then

$$
\begin{gathered}
d_{1}(x)=\frac{x}{2}\left(\frac{\sin x}{x}\right)^{\prime}, \quad d_{2}(x)=\frac{x^{3}}{2^{2}}\left(\frac{1}{x}\left(\frac{\sin x}{x}\right)^{\prime}\right)^{\prime}, \ldots, \\
d_{k}(x)=\frac{x^{2 k-1}}{2^{k}}\left(\frac{1}{x}\left(\frac{1}{x}\left(\cdots\left(\frac{1}{x}\left(\frac{\sin x}{x}\right)^{\prime}\right)^{\prime} \cdots\right)^{\prime}\right)^{\prime}\right)^{\prime}
\end{gathered}
$$

with $k$ th derivative, and

$$
d_{k+1}(x)=-k d_{k}(x)+\frac{x}{2} d_{k}^{\prime}(x) \quad(k=1,2,3, \ldots) .
$$


4 An identity related to Jordan's inequality

Hence $R_{k}=d_{k}(\pi / 2)$ with

$$
\begin{array}{cc}
R_{1}=d_{1}\left(\frac{\pi}{2}\right)=-\frac{1}{\pi}, & R_{2}=d_{2}\left(\frac{\pi}{2}\right)=\frac{12-\pi^{2}}{8 \pi}, \\
R_{3}=d_{3}\left(\frac{\pi}{2}\right)=\frac{-3\left(10-\pi^{2}\right)}{8 \pi}, & R_{4}=d_{4}\left(\frac{\pi}{2}\right)=\frac{\pi^{4}-180 \pi^{2}+1680}{128 \pi} .
\end{array}
$$

Also the above established identity (2.1) gives

$$
\begin{aligned}
\frac{\sin x}{x}= & \frac{2}{\pi}+\frac{1}{\pi^{3}}\left(\pi^{2}-4 x^{2}\right)+\frac{12-\pi^{2}}{16 \pi^{5}}\left(\pi^{2}-4 x^{2}\right)^{2} \\
& +\frac{10-\pi^{2}}{16 \pi^{7}}\left(\pi^{2}-4 x^{2}\right)^{3}+\frac{\pi^{4}-180 \pi^{2}+1680}{3072 \pi^{9}}\left(\pi^{2}-4 x^{2}\right)^{4} \\
& +\sum_{k=5}^{\infty} \frac{(-1)^{k} R_{k}}{k ! \pi^{2 k}}\left(\pi^{2}-4 x^{2}\right)^{k}
\end{aligned}
$$

for all $x>0$. Since $(-1)^{k} R_{k}>0(k=1,2,3, \ldots)$, we have the following corollary.

Corollary 2.2. If $0<x \leq \pi / 2$, then

$$
\begin{aligned}
\frac{\sin x}{x} \geq & \frac{2}{\pi}+\frac{1}{\pi^{3}}\left(\pi^{2}-4 x^{2}\right)+\frac{12-\pi^{2}}{16 \pi^{5}}\left(\pi^{2}-4 x^{2}\right)^{2} \\
& +\frac{10-\pi^{2}}{16 \pi^{7}}\left(\pi^{2}-4 x^{2}\right)^{3}+\frac{\pi^{4}-180 \pi^{2}+1680}{3072 \pi^{9}}\left(\pi^{2}-4 x^{2}\right)^{4}
\end{aligned}
$$

holds with equality if and only if $x=\pi / 2$. Furthermore, the constants $1 / \pi^{3},\left(12-\pi^{2}\right) /\left(16 \pi^{5}\right)$, $\left(10-\pi^{2}\right) /\left(16 \pi^{7}\right)$, and $\left(\pi^{4}-180 \pi^{2}+1680\right) /\left(3072 \pi^{9}\right)$ in (2.14) are the best.

The above established inequality (2.14) is much stronger than the left-hand side of inequality (1.5). Also one can add more positive terms to the right-hand side of inequality (2.14) to get higher accuracy.

Finally, it should be pointed out that, in order to give the right-hand side of inequality (1.4) or (1.5), the following Taylor expansion for $x / \sin x$ will play an important role as the above established identity (2.1) or (2.13).

Taylor expansion of $x / \sin x$.

$$
\begin{aligned}
\frac{x}{\sin x} & =\sum_{n=0}^{\infty}(-1)^{n+1} B_{2 n} \frac{2^{2 n}-2}{(2 n) !} x^{2 n} \\
& =1+\frac{1}{6} x^{2}+\frac{7}{360} x^{4}+\frac{31}{15120} x^{6}+\cdots \quad(|x|<\pi),
\end{aligned}
$$

where $B_{2 n}$ are the Bernoulli numbers satisfying $(-1)^{n+1} B_{2 n}>0(n=1,2,3, \ldots)$.

Recall that the Bernoulli numbers $B_{n}$ and the functions $B_{n}(x)$ are defined by

$$
\frac{t}{e^{t}-1}=\sum_{n=0}^{\infty} \frac{B_{n}}{n !} t^{n} \quad \frac{t e^{x t}}{e^{t}-1}=\sum_{n=0}^{\infty} \frac{B_{n}(x)}{n !} t^{n} \quad(|t|<2 \pi) .
$$


It is familiar that they have the following properties (see [3, Section I-13]):

$$
\begin{gathered}
B_{0}(x)=B_{0}=1, \quad B_{1}=\frac{-1}{2}, \quad B_{2}=\frac{1}{6}, \quad B_{4}=\frac{-1}{30}, \quad B_{6}=\frac{1}{42} ; \\
B_{2 n+1}=0, \quad(-1)^{n+1} B_{2 n}>0, \quad B_{n}(0)=B_{n} \quad(n \geq 1) ; \\
B_{n}(1-x)=(-1)^{n} B_{n}(x) \quad(n \geq 1) ; \\
B_{2 n}(x)=\frac{2(-1)^{n+1}(2 n) !}{(2 \pi)^{2 n}} \sum_{r=1}^{\infty} \frac{1}{r^{2 n}} \cos (2 \pi r x) \quad(n \geq 1,0 \leq x \leq 1)
\end{gathered}
$$

from which, we have

$$
B_{2 n-1}\left(\frac{1}{2}\right)=0, \quad 2^{2 n} B_{2 n}\left(\frac{1}{2}\right)=\left(2-2^{2 n}\right) B_{2 n} \quad(n \geq 1) .
$$

Therefore, for $|x|<\pi$,

$$
\begin{aligned}
\frac{x}{\sin x} & =\frac{2 x i e^{x i}}{e^{2 x i}-1}=\sum_{n=0}^{\infty} B_{n}\left(\frac{1}{2}\right) \frac{2^{n} i^{n}}{n !} x^{n} \\
& =\sum_{n=0}^{\infty} B_{2 n}\left(\frac{1}{2}\right) \frac{(-1)^{n} 2^{2 n}}{(2 n) !} x^{2 n}=\sum_{n=0}^{\infty}(-1)^{n+1} B_{2 n} \frac{2^{2 n}-2}{(2 n) !} x^{2 n} .
\end{aligned}
$$
ity:

From (2.15), we have the following type of strengthened right-hand Jordan's inequal-

$$
\frac{\sin x}{x} \leq\left(1+\frac{1}{6} x^{2}+\frac{7}{360} x^{4}+\frac{31}{15120} x^{6}\right)^{-1} \quad(|x|<\pi) .
$$

Also one can add more positive terms to the right-hand side of inequality (2.20) to get higher accuracy.

\section{Acknowledgment}

The author would like to thank the referees for their valuable suggestions.

\section{References}

[1] G. D. Anderson, S.-L. Qiu, M. K. Vamanamurthy, and M. Vuorinen, Generalized elliptic integrals and modular equations, Pacific Journal of Mathematics 192 (2000), no. 1, 1-37.

[2] L. Debnath and C.-J. Zhao, New strengthened Jordan's inequality and its applications, Applied Mathematics Letters 16 (2003), no. 4, 557-560.

[3] A. Erdélyi, Higher Transcental Functions, Vol. I, McGraw-Hill, New York, 1953.

[4] A. McD. Mercer, U. Abel, and D. Caccia, A sharpening of Jordan's inequality, The American Mathematical Monthly 93 (1986), 568-569.

[5] D. S. Mitrinović and P. M. Vasić, Analytic Inequalities, Springer, New York, 1970.

[6] A. Y. Özban, A new refined form of Jordan's inequality and its applications, Applied Mathematics Letters 19 (2006), no. 2, 155-160.

[7] S. Wu and L. Debnath, A new generalized and sharp version of Jordan's inequality and its application to the improvement of Yang Le inequality, Applied Mathematics Letters 19 (2006), no. 12, $1378-1384$. 
6 An identity related to Jordan's inequality

[8] _ A new generalized and sharp version of Jordan's inequality and its application to the improvement of Yang Le inequality II, to appear in Applied Mathematics Letters.

[9] L. Zhu, Sharpening Jordan's inequality and the Yang Le inequality, Applied Mathematics Letters 19 (2006), no. 3, 240-243.

[10] Sharpening Jordan's inequality and Yang Le inequality. II, Applied Mathematics Letters 19 (2006), no. 9, 990-994.

Jian-Lin Li: College of Mathematics and Information Science, Shaanxi Normal University, Xi' an 710062, China

E-mail address: jllimath@yahoo.com.cn 


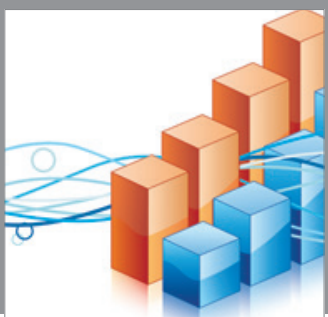

Advances in

Operations Research

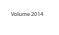

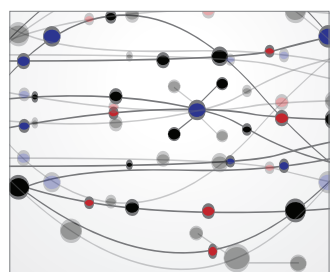

\section{The Scientific} World Journal
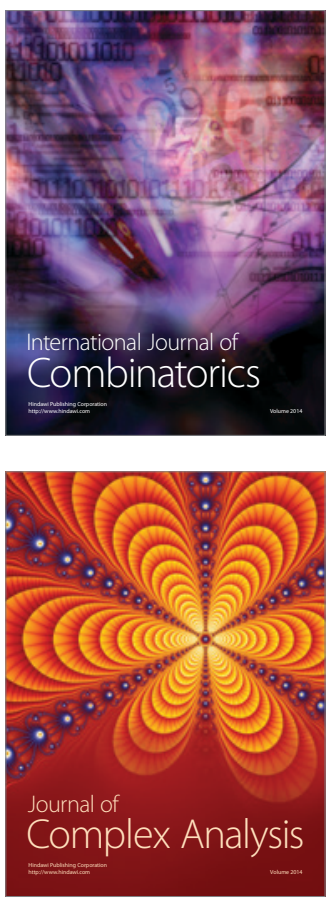

International Journal of

Mathematics and

Mathematical

Sciences
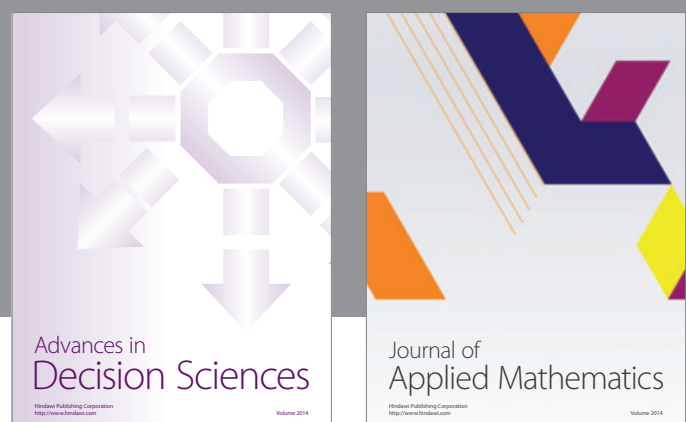

Journal of

Applied Mathematics
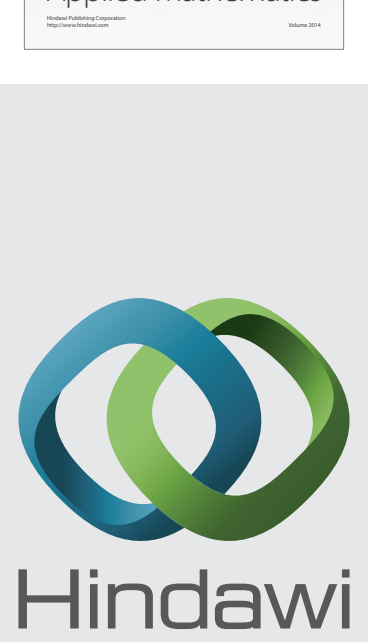

Submit your manuscripts at http://www.hindawi.com
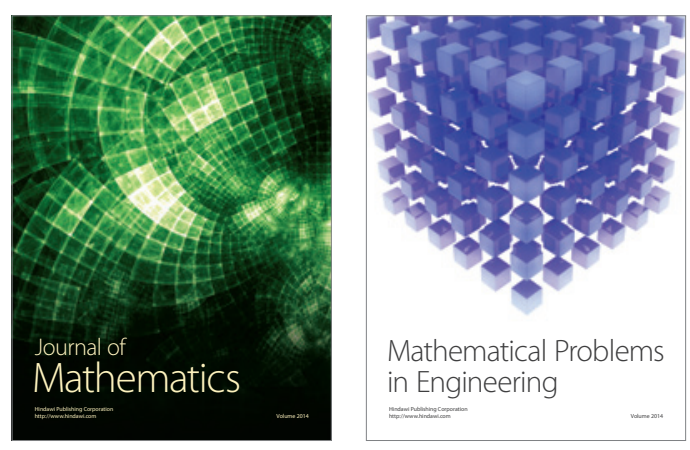

Mathematical Problems in Engineering
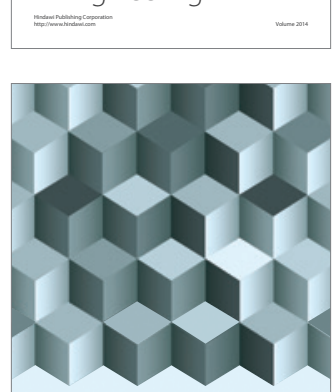

Journal of

Function Spaces
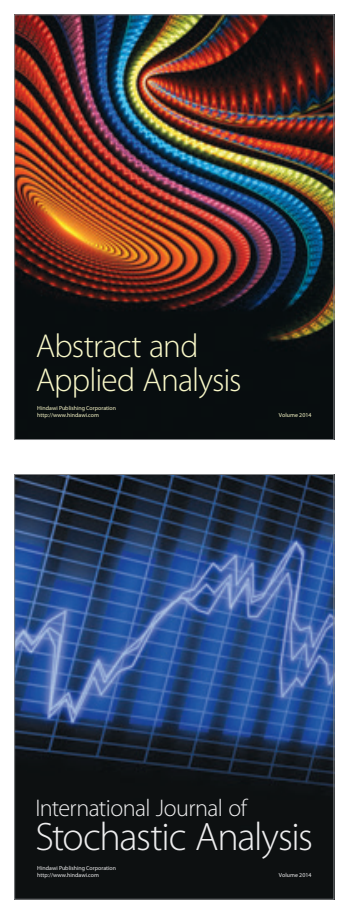

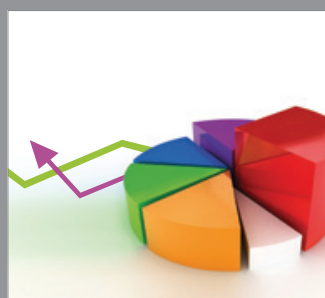

ournal of

Probability and Statistics

Promensencen
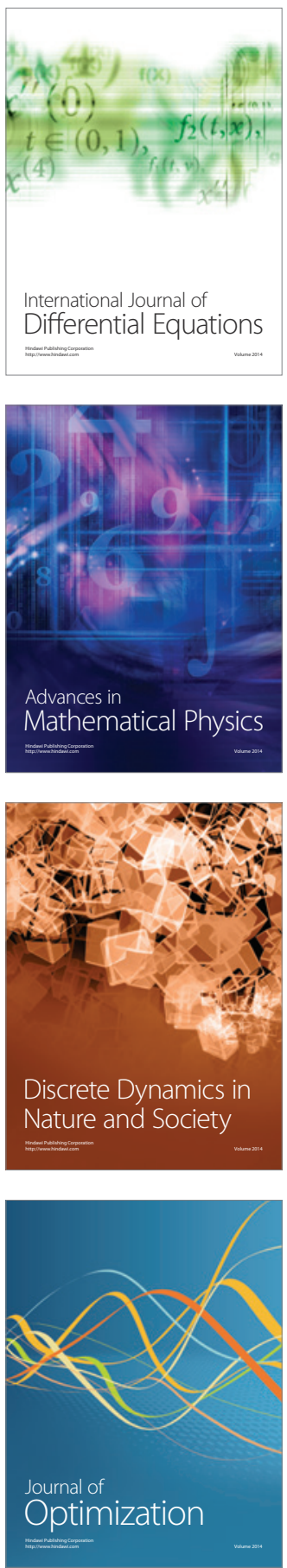